\title{
Antiplatelet Effects of Garlic and Chitosan: a Comparative Study between Fermented and Non-Fermented Preparations
}

\author{
Muhammad Irfan ${ }^{1, *}$, Minki Kim ${ }^{1, *}$, Hyuk-Woo Kwon ${ }^{2, * *}$, \\ Man Hee Rhee ${ }^{1, \dagger, * *}$ and Hyun-Kyoung Kim ${ }^{3, ;, * *}$ \\ ${ }^{1}$ Laboratory of Physiology and Cell Signaling, College of Veterinary Medicine, \\ Kyungpook National University, Daegu 41566, Korea \\ ${ }^{2}$ Department of Biomedical Laboratory Science, Far East University, Eumseong 27601, Korea \\ ${ }^{3}$ Department of Food Science and Engineering, Seowon University, Cheongju 28674, Korea
}

The incidence of cardiovascular diseases (CVDs) is increasing rapidly in developed countries, with CVDs now representing the leading cause of morbidity and mortality. Natural products and ethnomedicines have been shown to reduce the risk of CVDs. Garlic is a medicinal plant used throughout the world for its anti-inflammatory, antioxidant, and antiplatelet activities. Chitosan is a natural polysaccharide obtained from chitin, and derivatives of chitosan have been shown to inhibit platelet aggregation and adhesion. We hypothesized that fermented preparations of these products may possess stronger antiplatelet effects than the non-fermented forms owing to the increased bioavailability of the bioactive compounds produced during fermentation. Therefore, we compared these compounds via in vitro and ex vivo platelet aggregation assays by using standard light transmission aggregometry and ex vivo granule secretions from rat platelets. We found that fermented preparations exerted more potent and significant inhibition of platelet aggregation both in vitro and ex vivo. Likewise, ATP release from dense granules of platelets was also significantly inhibited in fermented preparation-treated rat platelets compared to that in non-fermented preparation-treated ones. We concluded that fermented preparations exerted more potent effects on platelet function both in vitro and ex vivo, possibly as a result of the increased bioavailability of active compounds produced during fermentation. We therefore suggest that fermented products may be potent therapeutics against platelet-related CVDs and can be used as antiplatelet and antithrombotic agents.

Key Words: Platelets, Fermented garlic, Fermented chitosan, Anti-platelet

Cardiovascular diseases (CVDs) are the leading cause of morbidity and mortality in developed countries (Maione et al., 2014). Multiple risk factors are involved in the pathophysiology, but platelets are considered the main etiological factor of CVDs. Platelet aggregation is a key step in the development and progression of atherosclerotic plaques responsible for narrowed blood vessels that may ultimately lead to stroke and heart attack (Michel et al., 2014). Platelet function can be suppressed pharmacologically, and this has been highly successful in decreasing thrombotic events;

Received: August 17, 2018 / Accepted: September 4, 2018

*Undergraduate student, ${ }^{* *}$ Professor.

${ }^{\dagger}$ Corresponding author: Man Hee Rhee. Laboratory of Physiology and Cell Signaling, College of Veterinary Medicine, Kyungpook National University, Daegu 41566, Korea.

Tel: +82-53-950-5967, Fax: +82-53-950-5955, e-mail: rheemh@knu.ac.kr

${ }^{\dagger}$ Corresponding author: Hyun-Kyoung Kim. Department of Food Science and Engineering, Seowon University, Chungbuk 28674, Korea.

Tel: +82-43-299-8474, Fax: +82-43-299-8470, e-mail: kimhk4@seowon.ac.kr

(C) The Korean Society for Biomedical Laboratory Sciences. All rights reserved.

(9)This is an Open Access article distributed under the terms of the Creative Commons Attribution Non-Commercial License (http://creativecommons.org/licenses/by-nc/3.0/) which permits unrestricted non-commercial use, distribution, and reproduction in any medium, provided the original work is properly cited. 
many clinically approved antiplatelet drugs are now available for the treatment of cardiovascular ailments. Unfortunately, as these drugs can lead to serious complications, such as gastric bleeding, and are ineffective in some patients (Barrett et al., 2008), there is a need for the development of more effective and safer approaches for the treatment and prevention of CVDs. One such approach includes the use of natural products with antithrombotic and anticoagulant activities (Rastogi et al., 2016). Ethnomedicine and natural products have been the focus of many recent studies as potential therapies for CVDs (Kerver et al., 2003), and several dietary and herbal compounds have been shown to contribute to a reduced risk of CVDs (Dutta-Roy, 2002).

Garlic has been shown to reduce cardiovascular diseases and its consumption confers significant cardioprotective effects to both animals and humans (Rahman and Billington, 2000; Banerjee and Maulik, 2002). Chitosan is a natural biodegradable and non-toxic cationic polysaccharide obtained from chitin. Its derivatives are known to inhibit platelet aggregation and adhesion (Jiang et al., 2012). For a long time, fermentation of foods has been appreciated owing to its significant commercial benefits, such as improved nutrient profile and enriched flavor. There are several health benefits of consuming fermented foods beyond the traditionally recognized effects on the digestive system (Kim et al., 2016). It was previously reported that fermentation enhances the quality and efficacy of food or herbal products through an improved nutrient profile (Lee et al., 2012). Therefore, we aimed to compare the effects of fermented garlic and chitosan with those of non-fermented compounds on platelet function.

For this purpose, collagen (native collagen fibrils (type I) from equine tendons), ADP, and thrombin were purchased from Chrono-log (Havertown, PA, USA). The ATP assay kit was obtained from the Biomedical Research Service Centre (Buffalo, NY, USA) and DMSO was acquired from SigmaAldrich (St. Louis, MO, USA). Water was obtained from J.T. Baker (Phillipsburg, NJ, USA). All chemicals were of reagent grade.

To prepare fermented chitosan, chitosan was first dissolved in hydrochloric acid and chitosanase was added to decompose the chitosan and produce chitosan oligosaccharide. To prepare fermented garlic, unpeeled garlic was placed in an autoclave and steamed to produce black garlic. Lactic acid bacteria were added for the second round of fermentation.

Male Sprague-Dawley (SD) rats (240 260 g) were purchased from Orient Co. (Seoul, Korea) and acclimatized for 1 week prior to the experiment in an animal room with controlled environmental conditions (12/12 h light/dark cycle, $23{ }^{\circ} \mathrm{C} \pm 2{ }^{\circ} \mathrm{C}$ temperature, and $50 \% \pm 10 \%$ humidity). The experiments were conducted in accordance with the IACUC guidelines and the protocols were approved by the Ethics Committee of College of Veterinary Medicine, Kyungpook National University, Daegu, Korea. The rats were randomly divided into six groups ( $\mathrm{n}=4$ in each group) for the ex vivo study and received a daily oral administration of vehicle, $300 \mathrm{mg} / \mathrm{kg}$ garlic (fermented and non-fermented), and 300 $\mathrm{mg} / \mathrm{kg}$ chitosan (fermented and non-fermented) for 7 days. Two hours after the final administration, blood samples were collected from rats for testing.

To prepare washed platelets, whole blood was collected from SD rats via heart puncture and transferred to a tube containing anticoagulant acid citrate dextrose solution. The blood was centrifuged at $170 \times g$ for 7 min to obtain plateletrich plasma (PRP), which was then centrifuged at $350 \times g$ for $7 \mathrm{~min}$ to isolate the washed platelets. The platelet concentration was adjusted to $3 \times 10^{8}$ cells $/ \mathrm{mL}$ by using Tyrode's buffer $(137 \mathrm{mM} \mathrm{NaCl}, 12 \mathrm{mM} \mathrm{NaHCO} 3,5.5 \mathrm{mM}$ glucose, $2 \mathrm{mM} \mathrm{KCl}, 1 \mathrm{mM} \mathrm{MgCl} 2$, and $1 \mathrm{mM} \mathrm{NaHPO}_{4}, \mathrm{pH}$ 7.4) for use in the platelet aggregation assays. All preparation procedures were performed at room temperature $\left(23^{\circ} \mathrm{C} \pm 2{ }^{\circ} \mathrm{C}\right)$.

Light-transmission aggregometry (Chrono-log, Havertown, PA, USA) was performed in accordance with a previously described method to assess platelet aggregation (Irfan et al., 2018). Briefly, the washed platelets were pre-incubated with various concentrations of either fermented garlic (F. garlic), non-fermented garlic (NF. garlic), fermented chitosan (F. chitosan), non-fermented chitosan (NF. chitosan), or vehicle for $2 \mathrm{~min}$ at $37^{\circ} \mathrm{C}$ in the presence of $1 \mathrm{mM}$ calcium chloride $\left(\mathrm{CaCl}_{2}\right)$, and then stimulated by the agonist collagen, ADP, or thrombin. The mixture was further incubated for $5 \mathrm{~min}$ with continuous stirring. To achieve ex vivo platelet aggregation, washed platelets obtained from vehicle-, F. garlic-, NF. garlic-, F. chitosan-, and NF. chitosan-treated rats were 
A

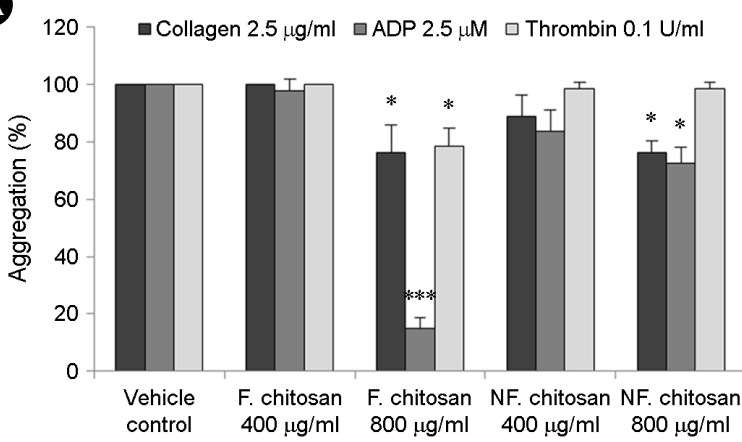

B

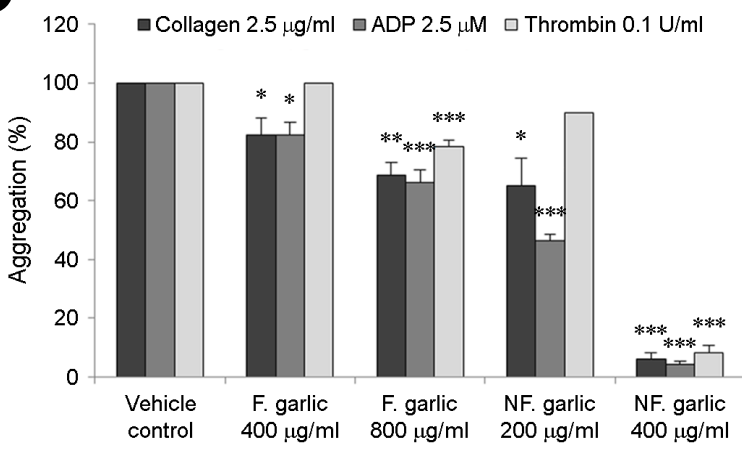

C

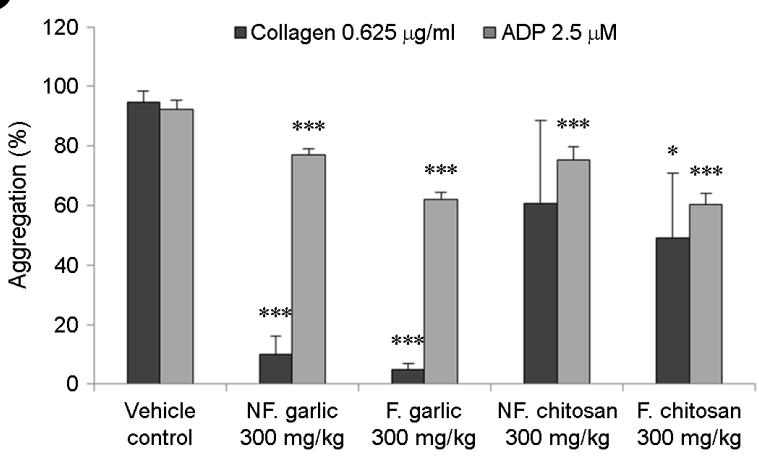

Fig. 1. Garlic and chitosan inhibit in vitro and ex vivo agoniststimulated platelet aggregation. (A-B) Washed platelets were pretreated with various concentrations of F. garlic, NF. garlic, F. chitosan, NF. Chitosan, or vehicle for $2 \mathrm{~min}$ in presence of $1 \mathrm{mM}$ $\mathrm{CaCl}_{2}$, and then stimulated with collagen, ADP, or thrombin for 5 min. (C) Washed platelets obtained from vehicle-, F. garlic-, NF. garlic-, F. chitosan-, or NF. chitosan-treated rats (all treatments at $300 \mathrm{mg} / \mathrm{kg}$ ) were incubated at $37^{\circ} \mathrm{C}$ with continuous stirring and then stimulated with collagen or ADP for $5 \mathrm{~min}$. The graph presents the mean \pm SEM of experiments performed on four independent days $\left(\mathrm{n}=1\right.$ each day). ${ }^{*} P<0.05, * * P<0.01$ and $* * * P<0.001$ compared with the vehicle control.

incubated at $37^{\circ} \mathrm{C}$ with continuous stirring and stimulated with collagen or ADP for $5 \mathrm{~min}$.

To determine the ex vivo effects of F. garlic, NF. garlic, F. chitosan, and NF. chitosan on dense granule secretion, an ATP assay was performed as previously described (Jeong et al., 2016). Briefly, washed platelets obtained from vehicle-, F. garlic-, NF. Garlic-, F. chitosan-, NF. Chitosan-treated groups were incubated at $37^{\circ} \mathrm{C}$ with continuous stirring and stimulated with collagen or ADP for $5 \mathrm{~min}$. The aggregation reaction was terminated and the platelet mixture was centrifuged. The ATP secretion of the obtained supernatant was determined by using an ATP assay kit (Biomedical Research Service Center) measured using a luminometer (GloMax 20/20, Promega, Madison, WI, USA).

The data were analyzed by using one-way analysis of variance (ANOVA) followed by Dunnett's post-hoc test to measure the statistical significance of the differences observed (SAS Institute Inc., Cary, NC, USA). All data are presented as the mean \pm standard error of the mean (SEM), and $P_{-}$ values $<0.05$ indicated statistical significance.

The in vitro effects of F. garlic, NF. garlic, F. chitosan, and NF. chitosan on platelet aggregation were assessed by using light transmission aggregometry. The effects of the compounds were tested against different ligands (collagen, ADP, and thrombin), and F. chitosan was found to be most effective against ADP-induced platelet aggregation, whereas F. garlic and NF. garlic were more potent and inhibited platelet aggregation against collagen, ADP, and thrombin (Fig. 1A-B). F. garlic and N.F. garlic markedly inhibited agonist-induced platelet aggregation in a dose-dependent manner.

To evaluate the ex-vivo effects, the rats were treated with $\mathrm{F}$. garlic, NF. garlic, F. chitosan, and NF. chitosan and the platelet aggregation assay was performed. Platelet aggregation was inhibited in all treatment groups unlike in the vehicletreated control group. Moreover, F. garlic and NF. garlic showed greater inhibition of collagen-induced platelet aggregation than of ADP-induced platelet aggregation (Fig. 1C).

Activated platelets release the contents of granules such as alpha granules and dense granules; this secretion of granule content enhances platelet activation, including intracellular signaling pathways.

The early phases of platelet activation are characterized by the rapid release of ATP (Jeong et al., 2017); therefore, we assessed collagen- and ADP-induced ATP secretion. As shown 


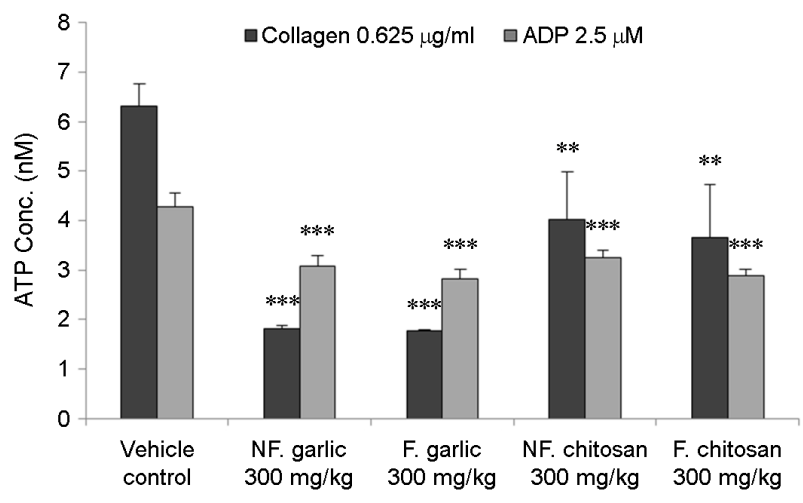

Fig. 2 Garlic and chitosan inhibit ex vivo ATP secretion. Washed platelets obtained from vehicle-, F. garlic-, NF. garlic-, F. chitosan-, or NF. chitosan-treated rats (all treatments at $300 \mathrm{mg} / \mathrm{kg}$ ) were incubated at $37^{\circ} \mathrm{C}$ with continuous stirring and stimulated with collagen or ADP for $5 \mathrm{~min}$. Following the termination of the platelet aggregation reaction, the concentration of ATP was assessed by using a luminometer. The results are presented as the mean \pm SEM of experiments performed on three independent days $(\mathrm{n}=1$ each day). ${ }^{* *} P<0.01$ and $* * * P<0.001$ compared with the vehicle control.

Fig. 2, F. garlic, NF. garlic, F. chitosan, and NF. chitosan significantly inhibited ATP release from dense granules in collagen- and ADP-stimulated platelets. Although both were strong inhibitors of ATP release, garlic appeared to be slightly more potent than chitosan (Fig. 2). These data suggest that the compounds exerted antiplatelet effects through the suppression of platelet aggregation and granule secretion.

In the present study, we evaluated the inhibitory effects of fermented and non-fermented preparations of garlic and chitosan on platelet aggregation and granule secretion. We found that fermented preparations significantly inhibited platelet aggregation in vitro and ex vivo and also inhibited ex-vivo ATP release from dense granules. Many studies have shown that platelets are continuously exposed to several factors that cause their activation and aggregation, such as collagen, ADP, thrombin, fibrinogen, von Willebrand factor (vWF), and thromboxane; however, inhibitory factors are also present, such as prostacyclin $\left(\mathrm{PGI}_{2}\right)$ and ADPase (Ruggeri and Mendolicchio, 2007). Any imbalance in these opposing factors may impair hemostasis; thus, a strong equilibrium is necessary for normal platelet function. Our results indicate that the pretreatment of platelets with garlic or chitosan preparations, especially fermented preparations, may help main- tain this balance and hemostasis. As fermentation enhances bioavailability and the absorption of food products (Lee et al., 2012; Rein et al., 2013; Kim et al., 2016), we concluded that the observed antiplatelet effects were a result of the increased bioavailability of bioactive compounds from fermented preparations.

\section{ACKNOWLEDGEMENT}

This research was supported by the Korean technology and information promotion agency (Industry-academyresearch cooperation technology development business of Assignment number C0563925).

\section{CONFLICT OF INTEREST}

No potential conflict of interest relevant to this article was reported.

\section{REFERENCES}

Banerjee SK, Maulik SK. Effect of garlic on cardiovascular disorders: A review. Nutrition Journal. 2002. 1: 4.

Barrett NE, Holbrook L, Jones S, Kaiser WJ, Moraes LA, Rana R, Sage T, Stanley RG, Tucker KL, Wright B, Gibbins JM. Future innovations in anti-platelet therapies. British Journal of Pharmacology. 2008. 154: 918-939.

Dutta-Roy AK. Dietary components and human platelet activity. Platelets. 2002. 13: 67-75.

Irfan M, Kwon T-H, Yun B-S, Park N-H, Rhee MH. Eisenia bicyclis (brown alga) modulates platelet function and inhibits thrombus formation via impaired P2Y12 receptor signaling pathway. Phytomedicine. 2018. 40: 79-87.

Jeong D, Irfan M, Kim S-D, Kim S, Oh J-H, Park C-K, Kim H-K, Rhee MH. Ginsenoside rg3-enriched red ginseng extract inhibits platelet activation and in vivo thrombus formation Journal of Ginseng Research. 2017. 41: 548-555.

Jeong D, Irfan M, Saba E, Kim S-D, Kim S-H, Rhee MH. Water soluble tomato concentrate regulates platelet function via the mitogen-activated protein kinase pathway. Korean Journal of Veterinary Research. 2016. 56: 67-74.

Jiang G-B, Lin Z-T, Xu X-J, Zhang H, Song K. Stable nanomicelles based on chitosan derivative: In vitro antiplatelet aggregation and adhesion properties. Carbohydrate Polymers. 2012. 88: 232-238. 
Kerver JM, Yang EJ, Bianchi L, Song WO. Dietary patterns associated with risk factors for cardiovascular disease in healthy us adults. The American Journal of Clinical Nutrition. 2003. 78: 1103-1110.

Kim B, Hong VM, Yang J, Hyun H, Im JJ, Hwang J, Yoon S, Kim JE. A review of fermented foods with beneficial effects on brain and cognitive function. Preventive Nutrition and Food Science. 2016. 21: 297.

Lee H-S, Kim M-R, Park Y, Park HJ, Chang UJ, Kim SY, Suh HJ. Fermenting red ginseng enhances its safety and efficacy as a novel skin care anti-aging ingredient: In vitro and animal study. Journal of Medicinal Food. 2012. 15: 1015-1023.

Maione F, De Feo V, Caiazzo E, De Martino L, Cicala C, Mascolo $\mathrm{N}$. Tanshinone iia, a major component of salvia milthorriza bunge, inhibits platelet activation via erk-2 signaling pathway. Journal of Ethnopharmacology. 2014. 155: 1236-1242.

Michel JB, Martin-Ventura JL, Nicoletti A, Ho-Tin-Noe B. Pathology of human plaque vulnerability: Mechanisms and consequences of intraplaque haemorrhages. Atherosclerosis. 2014. 234: 311-319.
Rahman K, Billington D. Dietary supplementation with aged garlic extract inhibits adp-induced platelet aggregation in humans. The Journal of Nutrition. 2000. 130: 2662-2665.

Rastogi S, Pandey MM, Rawat AK. Traditional herbs: A remedy for cardiovascular disorders. Phytomedicine. 2016. 23: 1082 -1089 .

Rein MJ, Renouf M, Cruz-Hernandez C, Actis-Goretta L, Thakkar SK, da Silva Pinto M. Bioavailability of bioactive food compounds: A challenging journey to bioefficacy. British Journal of Clinical Pharmacology. 2013. 75: 588-602.

Ruggeri ZM, Mendolicchio GL. Adhesion mechanisms in platelet function. Circulation Research. 2007. 100: 1673-1685.

https://doi.org/10.15616/BSL.2018.24.3.280

Cite this article as: Irfan M, Kim MK, Kwon HW, Rhee MH, Kim HK. Antiplatelet Effects of Garlic and Chitosan: a Comparative Study between Fermented and Non-Fermented Preparations. Biomedical Science Letters. 2018. 24: 280-284. 